\title{
Computing the long-distance contribution to second order weak amplitudes
}

\author{
Norman H. Christ* \\ Department of Physics, Columbia University, New York, NY 10025, USA \\ E-mail: nhc@phys.columbia.edu
}

\section{RBC and UKQCD collaborations}

The calculation of the long-distance contribution to the $K^{0}-\bar{K}^{0}$ mass matrix is divided into three parts: First, the calculation of the matrix element between kaon states of the product of two space-time integrated, $\Delta S=1$, four-quark weak operators. Second an RI/MOM subtraction to remove the short distance part of this matrix element in a fashion consistent with the calculation of the physical short distance part. Third an application of the Lellouch-Luscher method, generalized to second order in the weak interactions, to control finite volume errors. Such an approach promises to permit accurate lattice calculation of the $K_{L}-K_{S}$ mass difference and the long-distance contributions to $\varepsilon_{K}$.

The XXVIII International Symposium on Lattice Field Theory, Lattice2010

June 14-19, 2010

Villasimius, Italy

\footnotetext{
* Speaker.
} 


\section{Introduction}

Lattice QCD has been very successful at computing the effects of the electroweak interactions on the properties of the strongly interacting particles. For many processes the large mass of the $W^{ \pm}$ and $Z$ bosons cause their interactions with the quarks and gluons of the hadrons to take place in a very small space-time region. These short distance interactions can be evaluated using electroweak and QCD perturbation theory and their low energy effects on hadrons described by effective four quark operators. For example, this approach provides a good description of both first-order decays and even some second order processes such as the CP violating effects in $K^{0}-\bar{K}^{0}$ and $B^{0}-\bar{B}^{0}$ mixing. However, for general second order processes, in which two $W^{ \pm}$and/or $Z$ bosons appear, it is possible that while each $W^{ \pm}$or $Z$ exchange will appear to take place at a point, the points locating these two exchanges may be separated by a much larger distance $\sim 1 / \Lambda_{\mathrm{QCD}}$. Such long distance effects are believed to contribute to the $\mathrm{CP}$ violation seen in $K^{0}-\bar{K}^{0}$ mixing at the $5 \%$ level [1] but on at least the $20 \%$ level [2] for the CP conserving $K_{L}-K_{S}$ mass difference. ${ }^{1}$

Here we present a method to compute such long distance effects using lattice QCD, focused on the case of the $K_{L}-K_{S}$ mass difference. There are three complications which must be overcome. First we need to devise an Euclidean space expectation value which can be evaluated in lattice QCD and which contains the second order energy shift of interest.

Second, such a lattice quantity will involve a product of two, first-order weak Hamiltonian densities, $\mathscr{H}_{W}\left(x_{i}\right)_{i=1,2}$, each corresponding to one of the $W^{ \pm}$or $Z$ exchanges. The short distance behavior of this product as $\left|x_{1}-x_{2}\right| \rightarrow 0$ will not describe the actual behavior of the exchange of two $W^{ \pm}$or $Z$ bosons at nearby space-time points. Thus, this incorrect short distance behavior must be removed and replaced by the known, physical, short distance behavior described above.

Third, the effects of finite volume, necessary in a lattice calculation, must be removed. These appear especially significant since the infinite volume expression contains continuous integrals, often with vanishing energy denominators evaluated as principal parts, while the finite volume quantity is a simple sum of discrete finite volume states. Here a generalization of the method of Lellouch and Luscher [4] can be used. We will now discuss how each of these obstacles may be overcome in a calculation of the $K_{L}-K_{S}$ mass difference, $\Delta m_{K}$.

\section{Second order lattice amplitude}

The standard description of $K^{0}-\bar{K}^{0}$ mixing provides an expression for the $K_{L}-K_{S}$ mass difference which we will write as

$$
\Delta m_{K}=2 \mathscr{P} \sum_{\alpha} \frac{\left\langle\bar{K}^{0}\left|H_{W}\right| \alpha\right\rangle\left\langle\alpha\left|H_{W}\right| K^{0}\right\rangle}{m_{K}-E_{\alpha}} .
$$

Here $\mathrm{CP}$ violating effects, at the $0.1 \%$ level, have been neglected, we are summing over intermediate states $|\alpha\rangle$ with energy $E_{\alpha}$ and normalization factors associated with the conserved total momentum are suppressed. This generalized sum includes an integral over intermediate state energies and the $\mathscr{P}$ indicates the principal part of the integral over the $E_{\alpha}=m_{K}$ singularity.

\footnotetext{
${ }^{1}$ For a discussion of the lattice QCD calculation of long distance effects in different decay processes see Ref. [3].
} 
One possible way to capture a similar expression in a Euclidean space lattice calculation is to evaluate the time-integrated second-order product that if evaluated in Minkowski space would yield the $\Delta m_{K}$ contribution to the time evolution over a time interval $\left[t_{a}, t_{b}\right]$ :

$$
\mathscr{A}=\frac{1}{2}\left\langle\bar{K}^{0}\left(t_{f}\right) \int_{t_{a}}^{t_{b}} d t_{2} \int_{t_{a}}^{t_{b}} d t_{1} H_{W}\left(t_{2}\right) H_{W}\left(t_{1}\right) K^{0}\left(t_{i}\right)\right\rangle .
$$

Here the initial $K^{0}$ is created by a source $\bar{K}^{0}\left(t_{i}\right)$ at the time $t_{i}$ and the final $\bar{K}^{0}$ state destroyed by the sink $\bar{K}^{0}\left(t_{f}\right)$ at time $t_{f}$. This amplitude is represented schematically in Fig. 1. Equation 2.2 can be evaluated as a standard Euclidean space path integral with $t_{f} \gg t_{b} \gg t_{a} \gg t_{f}$. If the time extent of this Euclidean path integral is sufficiently large, then when converted to an operator expression, Eq. 2.2 becomes the vacuum expectation value of the time-ordered product of Heisenberg operators. Assuming that $t_{f}-t_{b}$ and $t_{a}-t_{i}$ are sufficiently large to project onto the $\bar{K}^{0}$ and $K^{0}$ states, substituting a sum over energy eigenstates $|n\rangle$, and integrating over $t_{2}$ and $t_{1}$ one obtains:

$$
\begin{aligned}
\mathscr{A}=- & \sum_{n \neq n_{0}} \frac{\left\langle\bar{K}^{0}\left|H_{W}\right| n\right\rangle\left\langle n\left|H_{W}\right| K^{0}\right\rangle}{m_{K}-E_{n}}\left\{t_{b}-t_{a}-\frac{e^{-\left(E_{n}-m_{K}\right)\left(t_{b}-t_{a}\right)}-1}{m_{K}-E_{n}}\right\} e^{-\left(t_{f}-t_{i}\right) m_{K}} \\
& -\frac{1}{2}\left(t_{b}-t_{a}\right)^{2}\left\langle\bar{K}^{0}\left|H_{W}\right| n_{0}\right\rangle\left\langle n_{0}\left|H_{W}\right| K^{0}\right\rangle e^{-\left(t_{f}-t_{i}\right) m_{K}} .
\end{aligned}
$$

Anticipating a result from Sec. 4, we have assumed that a single two-pion intermediate state $\left|n_{0}\right\rangle$ is degenerate with the kaon and treated that state separately in the time integrations.

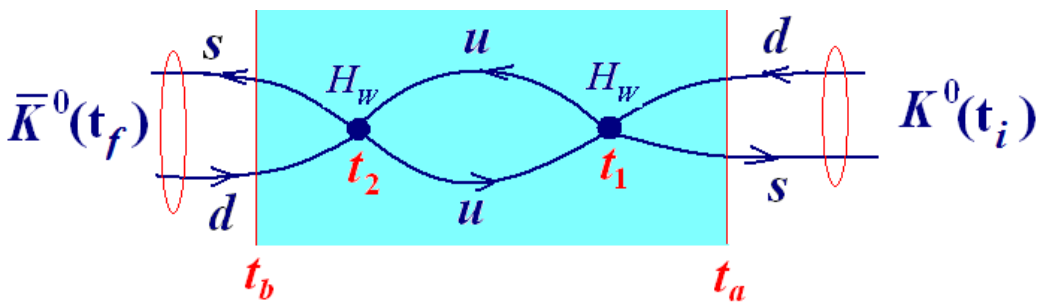

Figure 1: One type of diagram contributing to $\mathscr{A}$ of Eq. 2.2. Here $t_{2}$ and $t_{1}$ are integrated over the interval $\left[t_{a}, t_{b}\right]$, represented by the shaded region between the two vertical lines. In addition to this connected quark flow there will also be disconnected diagrams in which no quark lines connect $H_{W}\left(t_{2}\right)$ and $H_{W}\left(t_{1}\right)$.

The coefficient of the $\left(t_{b}-t_{a}\right)$ term in Eq. 2.3 is then a finite volume approximation to $\Delta m_{K}$ :

$$
\Delta m_{K}^{\mathrm{FV}}=2 \sum_{n \neq n_{0}} \frac{\left\langle\bar{K}^{0}\left|H_{W}\right| n\right\rangle\left\langle n\left|H_{W}\right| K^{0}\right\rangle}{m_{K}-E_{n}} .
$$

The other terms in Eq. 2.3 fall into four categories: i) The term independent of $t_{b}-t_{a}$ within the large curly brackets. This constant must be distinguished from the desired term proportional to $t_{b}-t_{a}$. ii) Exponentially decreasing terms coming from states $|n\rangle$ with $E_{n}>m_{K}$. These should be negligible if $t_{b}-t_{a}$ is sufficiently large. iii) Exponentially increasing terms coming from states $|n\rangle$ with $E_{n}<m_{K}$. These will be the dominant contributions and must be accurately determined and removed as discussed in the paragraph below ${ }^{2}$. iv) The final term proportional to $\left(t_{b}-t_{a}\right)^{2}$ arises because our choice of volume makes one $\pi-\pi$ state, $\left|n_{0}\right\rangle$, degenerate with the kaon.

\footnotetext{
${ }^{2}$ The author thanks Guido Martinelli and Stephen Sharpe for pointing out this behavior which had been overlooked when this talk was presented.
} 
The exponentially growing terms pose a significant challenge. Fortunately, we have some freedom to reduce their number and complexity. The two leading terms corresponding to the vacuum and single pion states can be computed separately and subtracted. Two pion states lying below $m_{K}$ can be eliminated using the same techniques that have been developed to evade the Maiani-Testa theorem and force the lowest energy $\pi-\pi$ state to be the on-shell $K \rightarrow \pi \pi$ decay product. Either choosing the kaon to have a non-zero laboratory momentum of $753 \mathrm{MeV}$ or introducing G-parity boundary conditions to force non-zero pion momentum can eliminate all $\pi-\pi$ states with energy below $m_{K}$, at least for those lattice volumes that will be accessible within the next few years.

\section{Short distance correction}

The product of operators appearing in Eq. 2.2 accurately describes the second order weak effects when the corresponding Hamiltonian densities $\mathscr{H}\left(x_{i}\right)_{i=1,2}$ are evaluated at space-time points separated by a few lattice units $a:\left|x_{2}-x_{1}\right| \gg a$. However, as $\left|x_{2}-x_{1}\right| \rightarrow 0$ the behavior is unphysical, being dominated by lattice artifacts rather than revealing the short distance structure of $W^{ \pm}$ and $Z$ exchange. Fortunately, non-perturbative Rome-Southampton methods can be applied here to accurately remove this incorrect behavior and replace it with the correct short distance behavior, that portion of the process that has been traditionally computed using lattice methods.

This can be done by identifying the short distance part of the amplitude by evaluating the four-quark, off-shell Green's function

$$
\Gamma_{\alpha \beta \gamma \delta}\left(p_{i}\right)=\left\langle\widetilde{\bar{d}}_{\alpha}\left(p_{4}\right) \widetilde{s}_{\beta}\left(p_{3}\right) \int d^{4} x_{1} d^{4} x_{2} \mathscr{H}_{W}\left(x_{2}\right) \mathscr{H}_{W}\left(x_{1}\right) \widetilde{s}_{\gamma}\left(p_{2}\right) \widetilde{\bar{d}}_{\delta}\left(p_{1}\right)\right\rangle .
$$

Here the quark fields are Fourier transformed and the gauge is fixed. A class of connected contributions to this Green's function is shown in Fig. 2. A standard application of Weinberg's theorem demonstrates that if the external momenta $p_{i}$ obey a condition such as $p_{i} \cdot p_{j}=\mu^{2}\left(1-4 \delta_{i j}\right)$, then for $\mu^{2} \gg \Lambda_{\mathrm{QCD}}^{2}$ all of the internal momenta contributing to $\Gamma\left(p_{i}\right)$ will have the scale $\mu$, up to terms of order $\Lambda_{\mathrm{QCD}}^{2} / \mu^{2}$.

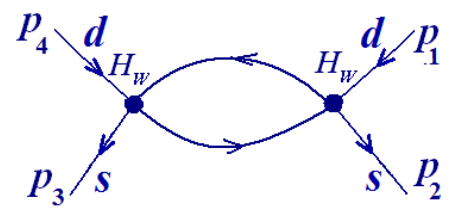

Figure 2: Diagram representing a class of connected contributions to the off-shell, four-quark Green's function defined in Eq. 3.1. In a non-perturbative evaluation of the Green's function $\Gamma\left(p_{i}\right)$, graphs of this sort including all possible gluon exchanges would be included.

At low energies this high momentum part of the integrated product $\mathscr{H}_{W}\left(x_{2}\right) \mathscr{H}_{W}\left(x_{1}\right)$ can be represented as a linear combination of four-quark operators $\left\{O_{s}\right\}_{1 \leq s \leq s}$. These operators are typically normalized by imposing conditions on off-shell Green's functions similar to that in Eq. 3.1 in which the product of $\mathscr{H}_{W}$ operators is replaced by $O_{s}$ and the same kinematic point evaluated. The result is an alternative expression for the short distance part of the amplitude $\mathscr{A}$ :

$$
\mathscr{A}_{\mathrm{SD}}=\left\langle\bar{K}^{0}\left(t_{f}\right) \int_{t_{a}}^{t_{b}} d x_{0} \int d^{3} x \sum_{s=1}^{S} c_{s}^{\mathrm{lat}}\left(\mu^{2}\right) O_{s}\left(\vec{x}, x_{0}\right) K^{0}\left(t_{i}\right)\right\rangle
$$


The functions $c_{s}^{\text {lat }}\left(\mu^{2}\right)$ are Wilson coefficients for the lattice-regularized operator product expanded in operators normalized using the regularization invariant (RI) Rome-Southampton scheme.

Thus, we can replace the incorrect short distance part of our lattice operator product by the correct continuum contribution by adding to the integrated operator product in Eq. 2.2 the operator:

$$
\int_{t_{a}}^{t_{b}} d x_{0} \int d^{3} x \sum_{s=1}^{S}\left\{c_{s}^{\mathrm{cont}}\left(\mu^{2}\right)-c_{s}^{\mathrm{lat}}\left(\mu^{2}\right)\right\} O_{s}\left(\vec{x}, x_{0}\right)
$$

Here the $\left\{c_{s}^{\text {cont }}\right\}_{1 \leq s \leq S}$ are the usual continuum Wilson coefficients that are computed from electroweak and QCD perturbation theory to represent the correct short distance part of the physical second order weak process while the lattice coefficients $c_{s}^{\text {lat }}$ can be computed from the somewhat elaborate but well defined lattice RI/MOM calculation of the Green's functions in Eq. 3.1.

An important issue on which the above argument depends is the degree to which the dimension6 , four-quark operators introduced above capture the entire short distance part of the lattice amplitude. Since the degree of divergence of the diagram shown in Fig. 2 is +2 , the integration that remains after the "subtraction" of the operator in Eq. 3.3 will still receive $O(1)$ contributions from the lattice scale. This difficulty can be avoided by including dimension eight terms in the Wilson expansion employed in Eq. 3.3. A more physical and more practical approach includes the charm quark in the lattice calculation so that GIM suppression makes the integration more convergent.

\section{Controlling finite volume errors}

We now turn to the heart of this proposal: a demonstration that the potentially large volume dependence coming from those energy denominators in Eq. 2.3 with $E_{n} \sim m_{K}$ can be removed, leaving $O\left(1 / L^{4}\right)$ finite-volume errors. This important conclusion is a consequence of a generalization of the original method of Lellouch and Luscher. The starting point is Luscher's relation [5] between an allowed, finite-volume, two-particle energy, $E=2 \sqrt{k^{2}+m_{\pi}^{2}}$ and the two-particle scattering phase shift $\delta(E)$ :

$$
\phi(k L / 2 \pi)+\delta(E)=n \pi
$$

where $n$ is an integer and the known function $\phi(q)$ is defined in Ref. [5].

Following Lellouch and Luscher we consider the $s$-wave, $\pi-\pi$ scattering phase shift as modified by the weak interactions and use Eq. 4.1 to connect this to the finite volume energies, determined using degenerate perturbation theory for the $K_{S} \leftrightarrow \pi-\pi$ finite volume system. For simplicity we will limit our discussion to the larger $\Delta I=1 / 2$ part of $H_{W}$ and the $I=0 \pi-\pi$ state. ${ }^{3}$ The relation between the finite and infinite volume second order mass shift is obtained by imposing Eq. 4.1, accurate through second order in $H_{W}$.

We begin by examining the energies, accurate through second order in the strangeness changing, $\Delta I=1 / 2$ weak Hamiltonian, $H_{W}$, of the finite volume system made up of a $K_{S}$ meson, an $I=0$ two-pion state $\left|n_{0}\right\rangle$ with energy $E_{n_{0}}$ nearly degenerate with $m_{K}$ and other single and multi-particle states coupled to $K_{S}$ and $\left|n_{0}\right\rangle$ by $H_{W}$. Following second order degenerate perturbation theory, we

\footnotetext{
${ }^{3}$ Treating the general case is not difficult: $H_{W}^{\Delta I=3 / 2} \cdot H_{W}^{\Delta I=3 / 2}$ and $H_{W}^{\Delta I=1 / 2} \cdot H_{W}^{\Delta I=1 / 2}$ can be analyzed in the same way while the combination $H_{W}^{\Delta I=1 / 2} \cdot H_{W}^{\Delta I=3 / 2}$ contains no two-pion intermediate states.
} 
can obtain the energies of the $K_{S}$ and two-pion state $\left|n_{0}\right\rangle$ as the eigenvalues of the $2 \times 2$ matrix:

$$
\left(\begin{array}{cc}
m_{K}+\sum_{n \neq n_{0}} \frac{\left|\left\langle n\left|H_{W}\right| K_{S}\right\rangle\right|^{2}}{m_{K}-E_{n}} & \left\langle K_{S}\left|H_{W}\right| n_{0}\right\rangle \\
\left\langle n_{0}\left|H_{W}\right| K_{S}\right\rangle & E_{n_{0}}+\sum_{n \neq K_{S}} \frac{\left|\left\langle n\left|H_{W}\right| n_{0}\right\rangle\right|^{2}}{E_{n_{0}}-E_{n}}
\end{array}\right) .
$$

Finite and infinite volume quantities can then be related by requiring that the eigenvalues of the $2 \times 2$ matrix in Eq. 4.2 solve Eq. 4.1 where the phase shift $\delta(E)$ is the sum of that arising from the strong interactions, $\delta_{0}(E)$, a resonant contribution from the $K_{S}$ pole and more familiar second-order Born terms:

$$
\delta(E)=\delta_{0}(E)+\arctan \left(\frac{\Gamma(E) / 2}{m_{K}+\Delta m_{K_{S}}-E}\right)-\pi \sum_{\beta \neq K_{S}} \frac{\left|\left\langle\beta\left|H_{W}\right| n_{0}\right\rangle\right|^{2}}{E-E_{\beta}} .
$$

Here $\Gamma(E)$ is proportional to the square of the $K_{S}$ - two pion vertex which becomes the $K_{S}$ width when evaluated at $E=m_{K}$ :

$$
\Gamma(E)=2 \pi\left|\left\langle\pi \pi(E)\left|H_{W}\right| K_{S}\right\rangle\right|^{2},
$$

where for the infinite volume, $I=0, s$-wave, 2-pion state we choose the convenient normalization $\left\langle\pi \pi(E) \mid \pi \pi\left(E^{\prime}\right)\right\rangle=\delta\left(E-E^{\prime}\right)$. The three terms in Eq. 4.3 are shown in Fig. 3.

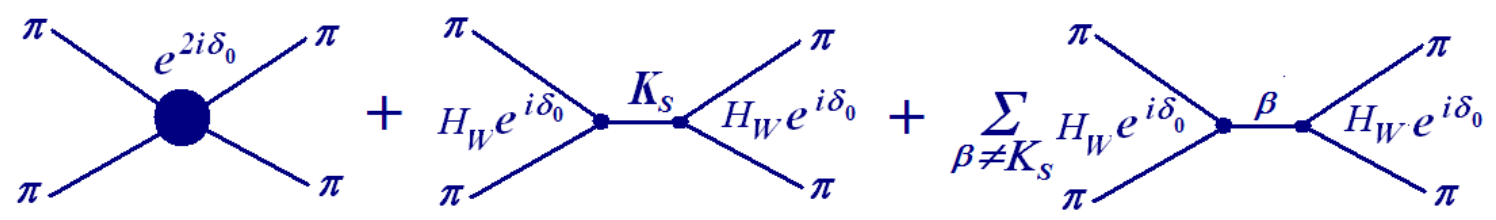

Figure 3: Diagrams showing the three contributions to the $\pi-\pi$ phase shift when both strong and second order weak effects are included. The states $\beta$ are multi-particle states with $S= \pm 1$.

The easiest case to examine is that in which the volume is chosen to make $E_{n_{0}}-m_{K}$ very small on the scale of $\Lambda_{\mathrm{QCD}}$ but large compared to $\Gamma$ or $\Delta m_{K}$, so that $m_{K}$ and $E_{n_{0}}$ are not "degenerate". Expanding Eq. 4.1 and the $\pi-\pi$ energy eigenvalue from Eq. 4.2 in $H_{W}$ and collecting all terms of second order in $H_{W}$ we find:

$$
\left.\frac{\partial\left(\phi+\delta_{0}\right)}{\partial E}\right|_{E=E_{n_{0}}}\left\{\frac{\left|\left\langle K_{S}\left|H_{W}\right| n_{0}\right\rangle\right|^{2}}{E_{n_{0}}-m_{K}}+\sum_{n \neq K_{S}} \frac{\left|\left\langle n\left|H_{W}\right| n_{0}\right\rangle\right|^{2}}{E_{n_{0}}-E_{n}}\right\}=\frac{\Gamma\left(E_{n_{0}}\right) / 2}{E_{n_{0}}-m_{K}}+\sum_{\beta \neq K_{S}} \frac{\pi\left|\left\langle\beta\left|H_{W}\right| \pi \pi\right\rangle\right|^{2}}{E_{n_{0}}-E_{\beta}}
$$

This relation has two useful consequences. First we can equate the residues of the kaon poles, $E_{n_{0}}=m_{K}$ on the left- and right-hand sides. This gives us the original Lellouch-Luscher relation. Second we can subtract the pole terms and equate the remaining parts of Eq. 4.5 evaluated at $E_{n_{0}}=m_{K}$. This second result will be used below to remove the second-order Born terms.

Finally, closer to the original spirit of Lellouch and Luscher, we substitute the phase shift $\delta(E)$ from Eq. 4.3 into Eq. 4.1 and require that the resulting equation be valid at the energy eigenvalues $E_{ \pm}$of the $2 \times 2$ matrix in Eq. 4.2 for a box chosen to make $E_{n_{0}}=m_{K}$. To zeroth order in $H_{W}$, this relation is the usual Luscher relation between $\delta_{0}(E)$ and the allowed, finite volume, $\pi-\pi$ energy. When Eq. 4.1 is expanded to first order, we reproduce the standard derivation of Lellouch 
and Luscher's relation. Expanding to second order in $H_{W}$ yields the desired relation between the finite and infinite volume expressions for the second order weak contribution to the $K_{S}$ difference:

$$
\begin{aligned}
\Delta m_{K_{S}}= & \sum_{n \neq n_{0}} \frac{\left|\left\langle n\left|H_{W}\right| K_{S}\right\rangle\right|^{2}}{m_{K}-E_{n}}+\frac{1}{\frac{\partial\left(\phi+\delta_{0}\right)}{\partial E}}\left[\frac{1}{2} \frac{\partial^{2}\left(\phi+\delta_{0}\right)}{\partial E^{2}}\left|\left\langle n_{0}\left|H_{W}\right| K_{S}\right\rangle\right|^{2}\right. \\
& \left.-\frac{\partial}{\partial E_{n_{0}}}\left\{\left.\frac{\partial\left(\phi+\delta_{0}\right)}{\partial E}\right|_{E=E_{n_{0}}}\left|\left\langle n\left|H_{W}\right| K_{S}\right\rangle\right|^{2}\right\}\right]
\end{aligned}
$$

where Eq. 4.5 , evaluated at $E_{n_{0}}=m_{K}$ with the pole terms subtracted has been used to eliminate the second-order Born terms. Note the $\partial / \partial E_{n_{0}}$ appearing in the final term in Eq. 4.6 must be evaluated by varying the spatial volume which determines $E_{n_{0}}{ }^{4}$

To obtain the $K_{L}-K_{S}$ mass difference we first observe that the $K_{L}$ second order mass shift is given by a formula similar to Eq. 4.6 in which $K_{L}$ replaces $K_{S}$ and all but the first term on the righthand side is omitted since $K_{L}$ does not couple to two pions, assuming CP conservation. Second if this new equation is subtracted from Eq. 4.6 the result is similar to Eq. 4.6 with $\Delta m=\Delta m_{K_{S}}-\Delta m_{K_{L}}$ on the left-hand side, the first term on the right-hand side is simply $\Delta m_{K}^{F V}$ of Eq. 2.4 and the remaining two $O\left(1 / L^{3}\right)$ correction terms on the right hand side of Eq. 4.6 are unchanged.

\section{Conclusion}

We have proposed a lattice method to compute the $K_{L}-K_{S}$ mass difference in which all errors can be controlled at the percent level. Both short and long distance effects are represented, including a possibly $\Delta I=1 / 2$-enhanced contribution from $I=0$ two pion states. Given the complexity of the analysis, the importance of using physical kinematics and the difficulty of the disconnected diagrams this calculation is not practical today but may be possible in the next few years.

The author thanks his RBC/UKQCD collaborators for important contributions to this work and Laurent Lellouch, Guido Martinelli and Stephen Sharpe for very helpful discussions. This work was supported in part by U.S. DOE grant DE-FG02-92ER40699.

\section{References}

[1] A. J. Buras, D. Guadagnoli and G. Isidori, On $\varepsilon_{K}$ beyond lowest order in the Operator Product Expansion, Phys. Lett. B688 (2010) 309-313 [arXiv:1002.3612 [hep-ph] ].

[2] S. Herrlich and U. Nierste, Enhancement of the $K(L)-K(S)$ mass difference by short distance $Q C D$ corrections beyond leading logarithms, Nucl. Phys. B419 (1994) 292-322 [hep-ph/9310311].

[3] G. Isidori, G. Martinelli and P. Turchetti, Rare kaon decays on the lattice, Phys. Lett. B633 (2006) 75-83 [hep-lat/0506026].

[4] L. Lellouch and M. Luscher, Weak transition matrix elements from finite-volume correlation functions, Commun. Math. Phys. 219 (2001) 31-44 [hep-lat/ 0003023 ].

[5] M. Luscher, Two particle states on a torus and their relation to the scattering matrix, Nucl. Phys. $\mathbf{B 3 5 4}$ (1991) 531-578.

\footnotetext{
${ }^{4}$ In a one-dimensional example, these derivative terms come naturally from a generalization of the usual contour integration relation between finite volume sums and infinite volume momentum integrals which includes the effects of a double pole arising from the vanishing on-shell energy denominator.
} 